\title{
Accurate calculation of the eigenvalues of non-uniform strings and membranes
}

Research Article

\author{
Paolo Amore ${ }^{1 *}$, Francisco M. Fernandez ${ }^{2 \dagger}$, Martin Rodriguez ${ }^{1 \neq}$ \\ 1 Facultad de Ciencias, CUICBAS, Universidad de Colima, \\ Bernal Díaz del Castillo 340, Colima, Colima, México \\ 2 INIFTA (UNLP, CCT La Plata-CONICET), División Química Teórica, \\ Blvd. 113 y $64(S / N)$, Sucursal 4, Casilla de Correo 16, \\ 1900 La Plata, Argentina
}

Received 15 January 2012; accepted 1 March 2012

\begin{abstract}
We describe a pseudospectral approach which combines a mapping procedure with the principle of minimal sensitivity to obtain accurate estimates for the eigenvalues of non-uniform strings and both uniform and nonuniform membranes. We illustrate the method on specific examples, including the case of a cross shaped membrane, with arms of infinite length.
\end{abstract}

PACS (2008): 02.60.-x, 02.70.-c, 02.70.Jn

Keywords: pseudospectral methods $\cdot$ Helmholtz equation

(c) Versita Sp. z o.o.

\section{Introduction}

This paper deals with the problem of obtaining precise numerical solutions to the Helmholtz equation, which governs the vibration modes of a classical object in one or two dimensions (a string or a membrane) ${ }^{1}$. In two dimensions, for the case of a membrane with variable density $\rho(x, y)$,

*E-mail: paolo.amore@gmail.com

${ }^{\dagger}$ E-mail: fernande@quimica.unlp.edu.ar

‡E-mail: martinalexander.rv@gmail.com

1 It also applies to the quantum mechanical problem of the Schrödinger equation of a free particle confined in a finite two dimensional region. the Helmholtz equation reads

$$
-\Delta \Psi_{n}(x, y)=E_{n} \rho(x, y) \Psi_{n}(x, y),
$$

where $E_{n}$ and $\Psi_{n}(x, y)$ are the eigenvalues and eigenfunctions of Eq. (1) respectively.

From a mathematical point of view both problems reduce to eigenvalue equations, which can be solved exactly only for a few special cases: the spectra of an uniform string or of a square or a circular membrane (just to mention two cases) are known exactly.

Most cases of practical interest cannot be solved exactly and one is forced to resort to alternative approaches that produce approximate solutions, either analytically or numerically. In this paper we focus on obtaining numerical solutions to those eigenvalue equations via a collocation (pseudospectral) approach. Typically in this approach one 
uses the interpolation properties of a given set of functions to derive a discretized version of the problem originally formulated in a continuum space. The approximate solutions are then found as eigenvalues and eigenvectors of a matrix whose dimension depends on the size of the set used for the collocation. The collocation approach is a convenient alternative to the widely used Rayleigh-Ritz method when the integrals have be carried out numerically (a time-consuming process). The reader may find a general introduction to pseudospectral methods in the remarkable book by Boyd [1] and several examples of their application to a variety of fields elsewhere [2-10].

Apart from these common features, the accuracy of a pseudospectral calculation depends on both the particular set of functions and their number. From a computational point of view it is desirable to keep the number of functions as small as possible, since it is directly related to the dimension of the collocation matrix and to the amount of computer memory required for its storage. Obviously, some sets of functions are more convenient than others for a given problem. For instance, Boyd et al. [10] compared the performance of the Fourier-sine mapped pseudospectral method [4] with the pseudospectral methods based on Laguerre functions or Chebyshev polynomials and concluded that the latter are preferable for the numerical solution of the problem of the hydrogen atom.

The approach that we describe in this paper is similar to the mapped Fourier method of Fattal et al. [4], where the efficiency of the uniform sampling of the Fourier grid is enhanced through a mapping of the coordinates. In their approach the appropriate map is found by allowing an optimal covering of the classical phase space associated with a given state. On the other hand, present strategy is motivated by the principle of minimal sensitivity (PMS) [11]. Here the map may depend on one or more artificial parameters (i.e. parameters which are not in the original problem) that would not affect the result if it were exact. In an approximate calculation there will be a residual dependence on such dummy parameters and we set their values so that their effect on the approximate result is minimal. This is the essence of the PMS [11].

The PMS has been used with remarkable success in many analytical calculations: one example is the "linear delta expansion" (LDE), which combines a perturbative expansion in an artificial parameter and the PMS for the physical observable, leading to accurate results [12-18].

It is our purpose to investigate whether the PMS enables us to obtain optimal grids with little computational effort. We compare our results with some others in the literature to test the efficiency of our approach.

Our paper is organized as follows: in Section 2 we describe the general collocation approach on non-uniform grids obtaining explicit matrix representations of the Hamiltonian operator in one and more dimensions; in Section 3 we describe a simple procedure which allows one to restore the variational nature of the collocation approach; in Section 4 we apply the method to several examples and compare our results with those available in the literature. Finally in Section 5 we draw conclusions.

\section{Collocation on arbitrary grids}

In this section we explicitly show how to build a set of "litthe sinc functions" (LSF) [19] that satisfy Dirichlet boundary conditions at the endpoints of a given finite coordinate interval. The generalization of these results to other boundary conditions is straightforward.

Let $\rho(x)$ be a positive function on the interval $x \in(-L, L)$ and define the functions

$$
\psi_{n}(x) \equiv \sqrt{\frac{2}{\sigma(L)}} \rho(x)^{1 / 4} \sin \left[n \pi \frac{\sigma(x)}{\sigma(L)}\right]
$$

where $n=1,2, \ldots$ and

$$
\sigma(x) \equiv \int_{-L}^{x} \sqrt{\rho(y)} d y
$$

These functions, which are closely related to the WKB solutions for a string of variable density (see, for example, page 490 in Ref. [20]), have been recently discussed by Amore [21]. By means of the change of variable $u=\sigma(x) / \sigma(L)$ it is straightforward to verify that these functions are orthonormal in $[-L, L]$ :

$$
\int_{-L}^{+L} \psi_{n}(x) \psi_{m}(x) d x=\delta_{n m}
$$

Since there is a one-to-one correspondence with the eigenfunctions of a quantum particle in a box the functions $\psi_{n}(x)$ span a complete set in that variable interval. For this reason we can follow the procedure of Amore et al. [19] and introduce the functions

$$
\bar{\delta}_{N}(x, y) \equiv C_{N} \sum_{n=1}^{N} \psi_{n}(x) \psi_{n}(y),
$$

where $C_{N}$ is independent of $x$ and $y$, although it depends on the number of terms in the sum. Its precise value will be determined later. It is not difficult to verify that 


$$
\begin{aligned}
\bar{\delta}_{N}(x, y) & =C_{N} \frac{\sqrt[4]{\rho(x) \rho(y)}}{\sigma(L)}\left[\frac{\sin \left(\frac{\pi N(\sigma(x)-\sigma(y))}{2 \sigma(L)}\right) \cos \left(\frac{\pi(N+1)(\sigma(x)-\sigma(y))}{2 \sigma(L)}\right)}{\sin \left(\frac{\pi(\sigma(x)-\sigma(y))}{2 \sigma(L)}\right)}\right. \\
& \left.-\frac{\sin \left(\frac{\pi N(\sigma(x)+\sigma(y))}{2 \sigma(L)}\right) \cos \left(\frac{\pi(N+1)(\sigma(x)+\sigma(y))}{2 \sigma(L)}\right)}{\sin \left(\frac{\pi(\sigma(x)+\sigma(y))}{2 \sigma(L)}\right)}\right]
\end{aligned}
$$

The function $\psi_{N}(x)$ in the last term of the sum exhibits the largest number of nodes $x_{k}(N), k=1, \ldots, N-1$ given by $\psi_{N}\left(x_{k}(N)\right)=0$ or

$$
\sigma\left(x_{k}(N)\right)=\frac{k}{N} \sigma(L)
$$

In some particular cases this equation may be solved exactly yielding explicit expressions for the $x_{k}(N)$. The simplest example is provided by a constant $\rho(x)$, which corresponds to the uniform grid already discussed in ref. [19]. If the exact solution of Eq. (5) is not possible we can calculate its roots numerically with little computational cost.

We choose the constant $C_{N}$ in such a way that it satisfies the condition $\bar{\delta}_{N}\left(x_{k}(N), x_{k}(N)\right)=1$ that leads to $N-1$ solutions of the form

$$
C_{N}^{(k)}=\frac{\sigma(L)}{N \sqrt{\rho\left(x_{k}(N)\right)}}, k=1,2, \ldots, N-1
$$

For simplicity we will write $x_{k}$ instead of $x_{k}(N)$ whenever there is no chance of ambiguity. When $\rho(x)=1$ we are led to the particular case of constant grid spacing $C_{N}=2 \mathrm{~L} / \mathrm{N}$ discussed earlier [19]. Actually, $C_{N}^{(k)}$ is roughly the grid spacing even in the general case. If we assume that $N \gg$ 1 , then we may approximately write $\frac{\sigma\left(x_{k}\right)-\sigma\left(x_{k-1}\right)}{x_{k}-x_{k-1}} \approx \sigma^{\prime}\left(x_{k}\right)=$ $\sqrt{\rho\left(x_{k}\right)}$, and, according to Eq. (5), we have $x_{k}-x_{k-1} \approx$ $\frac{\sigma(L)}{N} \frac{1}{\sqrt{\rho\left(x_{k}\right)}} \equiv C_{N}^{(k)}$, confirming our statement. It is worth noting that the grid spacing is finer where the density is larger, a property that is most valuable for building grids with an appropriate distribution of points.

By choosing the grid points $x_{k}$ as explained above we also obtain a second useful property of the LSF: $\bar{\delta}_{N}\left(x_{j}, x_{k}\right)=$ $0, j \neq k$ so that we finally have

$$
\bar{\delta}_{N}\left(x_{j}, x_{k}\right)=\delta_{k j}
$$

which is a generalization of a similar equation derived earlier for uniform grids [19].

We are now in a position to generalize the LSF developed earlier [19] and define what we may call the nonuniform Little Sinc Functions ( $v$ LSF):

$$
\begin{aligned}
& s_{k}(N, L, x) \equiv \bar{\delta}_{N}\left(x, x_{k}\right) \\
= & \frac{1}{2 N}\left[\frac{\rho(x)}{\rho\left(x_{k}\right)}\right]^{1 / 4}\left[\frac{\sin \left((2 N+1) \Delta_{-}\left(x, x_{k}\right)\right)}{\sin \left(\Delta_{-}\left(x, x_{k}\right)\right)}-\frac{\sin \left((2 N+1) \Delta_{+}\left(x, x_{k}\right)\right)}{\sin \left(\Delta_{+}\left(x, x_{k}\right)\right)}\right],
\end{aligned}
$$

where

$$
\Delta_{ \pm}\left(x, x_{k}\right) \equiv \frac{\pi\left(\sigma(x) \pm \sigma\left(x_{k}\right)\right)}{2 \sigma(L)}
$$

and $k=1,2, \ldots, N-1$. It is easy to verify that Eq. (8) reduces to Eq. (18) of Ref. [19] when $\rho(x)=1$ and $k \rightarrow$ $k-N / 2$ (note that $k=-N / 2+1, \ldots, N / 2-1$ in Ref. [19]). It is worth noting that the $s_{k}(N, L, x)$ is obtained from the

LSF on a uniform grid by means of the transformation $x \rightarrow \sigma(x)$ followed by multiplication by the factor $\left[\frac{\rho(x)}{\rho\left(x_{k}\right)}\right]^{1 / 4}$ which ensures orthonormality.

The $v$ LSF (8) may now be used to interpolate a given function $f(x)$ defined on the interval $[-L, L]$ and that vanishes 
at the endpoints $f( \pm L)=0$ in the following way:

$$
f(x) \approx \sum_{k=1}^{N-1} f\left(x_{k}\right) s_{k}(N, L, x)
$$

where $x_{k}=x_{k}(N)$ are the nonuniform grid points obtained earlier. We can justify Eq. (9) by writing the rhs as

$$
\sum_{k=1}^{N-1} f\left(x_{k}\right) s_{k}(N, L, x)=\sum_{n=1}^{N}\left[\sum_{k=1}^{N-1} C_{N}^{(k)} f\left(x_{k}\right) \psi_{n}\left(x_{k}\right)\right] \psi_{n}(x)
$$

where the term between parenthesis takes the form of a Riemann sum because $C_{N}^{(k)}$ is roughly the grid spacing as argued above. Thus, one obtains the well known decomposition of a function $f(x)$ in the basis set $\left\{\psi_{n}(x)\right\}$ :

$$
\begin{gathered}
\lim _{N \rightarrow \infty} \sum_{n=1}^{N}\left[\sum_{k=1}^{N-1} C_{N}^{(k)} f\left(x_{k}\right) s_{k}(N, L, x)\right] \psi_{n}(x) \\
=\sum_{n=1}^{\infty}\left[\int_{-L}^{L} f(x) \psi_{n}(x) d x\right] \psi_{n}(x) .
\end{gathered}
$$

The definition of the $v$ LSF given above enables us to generalize another property of the LSF:

$$
\int_{-L}^{+L} s_{k}(N, L, x) s_{j}(N, L, x) d x=\frac{\sigma(L)}{N \sqrt{\rho\left(x_{k}\right)}} \delta_{k j}
$$

which for constant density reduces to Eq.(20) of Ref. [19]. From the approximate interpolation expression (9) we also obtain that

$$
\int_{-L}^{+L} f(x) s_{k}(N, L, x) d x \approx \frac{\sigma(L)}{N \sqrt{\rho\left(x_{k}\right)}} f\left(x_{k}\right)
$$

which justifies the interpretation of $s_{k}(N, L, x) / C_{N}^{(k)}$ as an approximate Dirac delta function on the grid.

In order to obtain a suitable matrix representation for the kinetic-energy operator $\hat{T}=-\frac{d^{2}}{d x^{2}}$ it is convenient to consider the hermitian operator

$$
\hat{O}=-\frac{1}{\sqrt{\rho(x)}} \frac{d^{2}}{d x^{2}} \frac{1}{\sqrt{\rho(x)}}
$$

which naturally appears in the study of the vibrations of a string with variable density $[22,23]$. In the present case, however, $\rho(x)$ is not a physical density but a mathematical tool for producing a non-uniform grid of points. Upon operating with $\hat{O}$ on a basis function $\psi_{n}(x)$ given by Eq. (2) we obtain

$$
\hat{O} \psi_{n}(x)=\left[\frac{n^{2} \pi^{2}}{\sigma(L)^{2}}-\frac{5 \rho^{\prime}(x)^{2}-4 \rho(x) \rho^{\prime \prime}(x)}{16 \rho(x)^{3}}\right] \psi_{n}(x) .
$$

This equation suggests that $\psi_{n}(x)$ tends to become an eigenfunction of $\hat{O}$ when $n \rightarrow \infty$. In this limit the WKB solutions, $\psi_{n}^{(W K B)}(x)=\psi_{n}(x) / \sqrt{\rho(x)}$ tend to the solutions of the corresponding inhomogeneous Helmholtz equation. This property has already been exploited in Ref. [21] to obtain a perturbative expansion around that basis set. It was pointed out in Ref. [21] that in certain particular cases the functions $\psi_{n}(x)$ are exact eigenfunctions to the problem. An example is provided by the density $\rho(x)$ that obeys the differential equation

$$
5 \rho^{\prime}(x)^{2}-4 \rho(x) \rho^{\prime \prime}(x)=16 \kappa \rho^{3}(x)
$$

with $\kappa$ an arbitrary constant.

The general solution to this equation is of the form

$$
\rho(x)=\frac{256 c_{1}^{2}}{\left(c_{1}^{2}\left(c_{2}+x\right)^{2}+256 \kappa\right)^{2}}
$$

where $c_{1}$ and $c_{2}$ are constants of integration. When $k=0$ this expression reduces to the problem discussed long time ago by Borg [24], which is isospectral to a homogeneous string (see, for example, Ref. [25]).

Another example of string with a density satisfying Eq. (11) is the one discussed by Lord Rayleigh [26] which has recently been studied by Gottlieb (see Eq. (4.7) of Ref. [25], which corresponds to the form given in Eq. (12), after setting $k=0$ ). Gottlieb also identifies a string which is isospectral to the Rayleigh one in terms of a transformation that leaves the spectrum invariant.

The operator

$$
\hat{S}=-\frac{1}{\sqrt{\rho(x)}} \frac{d^{2}}{d x^{2}} \frac{1}{\sqrt{\rho(x)}}+\frac{5 \rho^{\prime}(x)^{2}-4 \rho(x) \rho^{\prime \prime}(x)}{16 \rho(x)^{3}},
$$

is diagonal in the basis set $\left\{\psi_{n}(x)\right\}$ because

$$
\hat{S} \psi_{n}(x)=\frac{n^{2} \pi^{2}}{\sigma(L)^{2}} \psi_{n}(x)
$$

We want to calculate the matrix elements of the operator $\hat{S}$ in the basis set of normalized $v \operatorname{LSF} s_{k}(x) / \sqrt{C_{N}^{(k)}}$ :

$$
S_{k l}=\frac{1}{\sqrt{C_{N}^{(k)} C_{N}^{(l)}}} \int_{-L}^{L} s_{k}(x) \hat{S} s_{l}(x) d x
$$


For convenience we also normalize the density with the condition $\sigma(L)=2 \mathrm{~L}$, which maps the original interval $[-L, L]$ onto a region of the same length $[0,2 L]$. Since $\psi_{N}\left(x_{k}\right)=0$ then we can write

$$
s_{k}(x)=C_{N}^{(k)} \sum_{n=1}^{N-1} \psi_{n}(x) \psi_{n}\left(x_{k}\right)
$$

and

$$
\begin{aligned}
S_{k l} & =\sqrt{C_{N}^{(k)} C_{N}^{(l)} \sum_{n=1}^{N-1} \psi_{n}\left(x_{k}\right) \psi_{n}\left(x_{l}\right) \frac{n^{2} \pi^{2}}{\sigma(L)^{2}}} \\
& =\frac{1}{\left(\rho\left(x_{k}\right) \rho\left(x_{l}\right)\right)^{1 / 4}} \frac{1}{N \sigma(L)} \sum_{n=1}^{N-1} \psi_{n}\left(x_{k}\right) \psi_{n}\left(x_{l}\right) n^{2} \pi^{2} \\
& =-c_{k l}^{(2)},
\end{aligned}
$$

where

$$
c_{k l}^{(2)}=\left\{\begin{array}{cl}
-\frac{\pi^{2}\left(-3 \csc ^{2}\left(\frac{\pi k}{N}\right)+2 N^{2}+1\right)}{24 L^{2}}, & k=l \\
-\frac{\pi^{2} \cos (\pi(k+l)) \sin \left(\frac{\pi k}{N}\right) \sin \left(\frac{\pi l}{N}\right)}{2 L^{2}\left(\cos \left(\frac{\pi k}{N}\right)-\cos \left(\frac{\pi l}{N}\right)\right)^{2}}, & k \neq l
\end{array} .\right.
$$

The matrix elements $c_{k l}^{(2)}$ agree with the matrix elements for the second derivative given in Ref. [19] after substituting $k \rightarrow k+N / 2$ and $l \rightarrow l+N / 2$.

The calculation of the matrix elements of the operator $\hat{D} \equiv-\frac{5 \rho^{\prime}(x)^{2}-4 \rho(x) \rho^{\prime \prime}(x)}{16 \rho(x)^{2}}$ is straightforward if we take into consideration the interpolation properties of the $v$ LSF:

$$
\begin{aligned}
D_{k l} & =\frac{1}{\sqrt{C_{N}^{(k)} C_{N}^{(l)}}} \int_{-L}^{L} s_{k}(x) \hat{D} s_{l}(x) d x \\
& \approx-\frac{5 \rho^{\prime}\left(x_{k}\right)^{2}-4 \rho\left(x_{k}\right) \rho^{\prime \prime}\left(x_{k}\right)}{16 \rho\left(x_{k}\right)^{2}} \delta_{k l}
\end{aligned}
$$

These results enable us to derive an expression for the matrix elements of the kinetic-energy operator:

$$
T_{k l}=\left[-\frac{d^{2}}{d x^{2}}\right]_{k l}=\sqrt{\rho\left(x_{k}\right)} S_{k l} \sqrt{\rho\left(x_{l}\right)}+D_{k l}
$$

We observe that

- For a constant $\rho(x)$ this matrix reduces to the one derived earlier by Amore et al. [19].

- The matrix for the operator $\hat{S}$ is universal; i.e. it is not specific to a given problem and therefore it can be calculated once for a given grid and stored.

- The matrices for $\hat{D}$ and $\sqrt{\rho}$ are specific to the problem under consideration and therefore they need to be calculated each time; however they are diagonal and such calculation is fast and efficient.

- The collocation on the grid does not require the calculation of integrals.

- All the matrix elements can be obtained with arbitrary precision.

The matrix representation of the second derivative is suitable for the approximate calculation of the eigenvalues and eigenfunctions of the Helmholtz and Schrödinger equations and we discuss specific applications in the following sections ${ }^{2}$.

The present approach is equivalent to the one proposed some time ago by Guardiola and Fernández [27] who applied it to the one-dimensional Schrödinger equation

$$
-\frac{d^{2} \Psi}{d r^{2}}+V(r) \Psi(r)=E \Psi(r)
$$

where $r \in(a, b), \Psi(a)=\Psi(b)=0$ and $a$ and $b$ can be either finite or infinite. Under the change of variable $r=r(x)$, where $r(x)$ is a monotonously increasing function of $x$, Eq. (14) is transformed into the equivalent equation

$$
-\frac{1}{G^{2}(x)} \frac{d^{2}}{d x^{2}} \frac{1}{G^{2}(x)} \Phi(x)+\left[V(r(x))-\frac{G^{\prime \prime}(x)}{G(x)^{5}}+2 \frac{G^{\prime}(x)^{2}}{G(x)^{6}}\right] \Phi(x)=E \Phi(x)
$$

where $G(x)=\sqrt{r^{\prime}(x)}$. Setting $G(x)=\rho(x)^{1 / 4}$ this equation reduces to

$$
-\frac{1}{\sqrt{\rho(x)}} \frac{d^{2}}{d x^{2}} \frac{1}{\sqrt{\rho(x)}} \Phi(x)+\left[V(r(x))+\frac{5 \rho^{\prime}(x)^{2}-4 \rho(x) \rho^{\prime \prime}(x)}{16 \rho(x)^{3}}\right] \Phi(x)=E \Phi(x),
$$


and the connection between both approaches is evident. However, the point of view is different because in the first case one does not transform the equation but generates a non-uniform grid, while in the second case one keeps the grid uniform and transforms the equation.

In what follows we briefly show how to apply the same approach to the Schrödinger equation in $d$ dimensions. We assume that the Hamiltonian operator is of the form

$$
\begin{aligned}
\hat{H} & =-\frac{1}{2} \Delta_{d}+V\left(x_{1}, \ldots, x_{d}\right) \\
\Delta_{d} & =\frac{\partial^{2}}{\partial x_{1}^{2}}+\cdots+\frac{\partial^{2}}{\partial x_{d}^{2}}
\end{aligned}
$$

In this case we may, for example, resort to $d$ density functions $\rho_{1}\left(x_{1}\right), \ldots, \rho_{d}\left(x_{d}\right)$ and obtain the matrix elements for the Laplacian operator as

$$
\begin{aligned}
& {\left[-\Delta_{d}\right]_{k_{1}, \ldots, k_{d} ; l_{1}, \ldots, l_{d}} } \\
= & {\left[\sqrt{\rho\left(x_{k_{1}}\right)} S_{k_{1} l_{1}} \sqrt{\rho\left(x_{l_{1}}\right)}+D_{k_{1} l_{1}}\right] \delta_{k_{2} l_{2}} \ldots \delta_{k_{d} l_{d}}+\ldots } \\
+ & {\left[\sqrt{\rho\left(x_{k_{d}}\right)} S_{k_{d} l_{d}} \sqrt{\rho\left(x_{l_{d}}\right)}+D_{k_{d} l_{d}}\right] \delta_{k_{1} l_{1}} \ldots \delta_{k_{d-1} l_{d-1}}(17) }
\end{aligned}
$$

The matrix elements for the potential-energy operator take a somewhat simple form: $[V]_{k_{1}, \ldots, k_{d} ; l_{1}, \ldots, l_{d}}=$
$V\left(x_{k_{1}}, \ldots, x_{k_{d}}\right) \delta_{k_{1} l_{1}} \delta_{k_{2} l_{2}} \ldots \delta_{k_{d} l_{d}}$.

These expressions arise from a basis set of $v$ LSF constructed as products of one-dimensional $v L S F$ : $s_{k_{1}}\left(N_{1}, L_{1}, x_{1}\right) \ldots s_{k_{d}}\left(N_{d}, L_{d}, x_{d}\right)$. Besides, the $j^{\text {th }}$ coordinate of a point of the $d$-dimensional grid is obtained by means of the $j^{\text {th }}$ density $\rho_{j}\left(x_{j}\right)$, exactly as indicated above for the one-dimensional case.

The collocation matrix for the laplacian is sparse. If there are $N-1$ grid points in every spatial dimension, the number of nonzero matrix elements is $d(N-1)^{d+1}$ over a total of $(N-1)^{2 d}$ elements.

Note that present approach applies straightforwardly to more complicated forms of the Hamiltonian operator, such as

$$
\hat{H}=-\frac{1}{2} \frac{1}{\sqrt{\sum\left(x_{1}, \ldots, x_{d}\right)}} \Delta_{d} \frac{1}{\sqrt{\sum\left(x_{1}, \ldots, x_{d}\right)}},
$$

which appears in the solution of the Helmholtz equation in an inhomogeneous medium with density $\Sigma\left(x_{1}, \ldots, x_{d}\right)$. In two dimensions $\Sigma\left(x_{1}, x_{2}\right)$ may be the conformal density obtained when an arbitrary domain is mapped onto a square one $[22,28]$. In such a case the matrix representation of $\hat{H}$ on the arbitrary grid will be

$$
\begin{aligned}
& \frac{1}{2} \frac{1}{\sqrt{\sum\left(x_{k_{1}}, \ldots, x_{k_{d}}\right)}}\left[-\Delta_{d}\right]_{k_{1}, \ldots, k_{d} ; l_{1}, \ldots, l_{d}} \frac{1}{\sqrt{\sum\left(x_{l_{1}}, \ldots, x_{l_{d}}\right)}} \\
= & \frac{1}{2} \frac{1}{\sqrt{\sum\left(x_{k_{1}}, \ldots, x_{k_{d}}\right)}}\left\{\left[\sqrt{\rho\left(x_{k_{1}}\right)} S_{k_{1} l_{1}} \sqrt{\rho\left(x_{l_{1}}\right)}+D_{k_{1} l_{1}}\right] \delta_{k_{2} l_{2}} \ldots \delta_{k_{d} l_{d}}+\ldots\right. \\
+ & {\left.\left[\sqrt{\rho\left(x_{k_{d}}\right)} S_{k_{d} l_{d}} \sqrt{\rho\left(x_{l_{d}}\right)}+D_{k_{d} l_{d}}\right] \delta_{k_{1} l_{1}} \ldots \delta_{k_{d-1} l_{d-1}}\right\} \frac{1}{\sqrt{\sum\left(x_{l_{1}}, \ldots, x_{l_{d}}\right)}} }
\end{aligned}
$$

Note that the matrix containing the physical (or conformal) density is diagonal.

\section{Improved collocation}

Each $s_{k}(N, L, x)$ is a linear combination of $\psi_{1}, \psi_{2}, \ldots, \psi_{N-1} ;$ therefore the basis sets $\left\{s_{k}\right\}_{k=1}^{N-1}$ and $\left\{\psi_{k}\right\}_{k=1}^{N-1}$ span the same vector subspace. For this reason each $s_{k}(N, L, x)$ can be exactly written as a linear combination of the functions in the set $\left\{s_{k}(M, L, x)\right\}_{k=1}^{M-1}$, provided that $M>N$. For convenience in what follows we resort to the bra-ket notation $|N, k\rangle$ meaning that $s_{k}(N, L, x)=\langle x \mid N, k\rangle \sqrt{C_{N}^{(k)}}$.

We assume that the Hamiltonian operator is of the form
$\hat{H}=\hat{T}+\hat{V}$. It is not difficult to calculate the matrix elements $T_{i, j}^{(N)}=\langle i, N|\hat{T}| N, j\rangle$ by means of the LSF as discussed above. However, the matrix elements $V_{i, j}^{(N)}=$ $\langle i, N|\hat{V}| N, j\rangle$ may not be so easily calculated and for that reason we have resorted to the following property of the LSF:

$$
\lim _{N \rightarrow \infty} V_{i, j}^{(N)}=V_{i} \delta_{i, j}
$$

For practical purposes we have carried out the approximation $V_{i, j}^{(N)} \approx V_{i} \delta_{i, j}$ which we have assumed to be valid if $N$ is large enough. However, it has been pointed out 
that in some cases this approximation may not particularly good [5].

When we diagonalize the Hamiltonian matrix $H_{i, j}^{(N)}=$ $T_{i, j}^{(N)}+V_{i} \delta_{i, j}$ the approximate eigenvalues may not be upper bounds to the actual ones because of the use of approximate matrix elements $V_{i, j}^{(N)}$. We may proceed in a different way and define the projection operators

$$
\hat{P}_{N}=\sum_{k=1}^{N-1}|N, k\rangle\langle k, N|
$$

According to what was stated above it is obvious that $\hat{P}_{N} \hat{P}_{M}=\hat{P}_{M} \hat{P}_{N}=\hat{P}_{N}$ for all $M>N$.

Since $\hat{P}_{M}|N, k\rangle=|N, k\rangle$ then we have

$$
\langle i, N|\hat{H}| N, j\rangle=\langle i, N|\hat{T}| N, j\rangle+\left\langle i, N\left|\hat{P}_{M} \hat{V} \hat{P}_{M}\right| N, j\right\rangle
$$

If $M$ is large enough we may carry out the approximation

$$
\hat{P}_{M} \hat{V} \hat{P}_{M} \approx \sum_{k=1}^{M-1}|M, k\rangle V_{k}\langle k, M|
$$

so that

$$
V_{i, j}^{(N)} \approx \sum_{k=1}^{M-1}\langle i, N \mid M, k\rangle V_{k}\langle k, M \mid N, j\rangle
$$

If $M$ is large enough we may recover the variational properties of the Rayleigh-Ritz method because we have a better representation of the operator $\hat{V}$ and its matrix elements.

The matrix $\Delta$ with elements $\Delta_{i, j}=\left\langle i, M\left|\hat{P}_{N}\right| M, j\right\rangle$ satisfies

$$
\Delta^{2}=\Delta, \operatorname{tr}(\Delta)=N-1
$$

We can formally write $\hat{P}_{N}<\hat{P}_{M}<\hat{1}$ in the sense that $\left\langle\psi\left|\hat{P}_{N}\right| \psi\right\rangle\left\langle\left\langle\psi\left|\hat{P}_{M}\right| \psi\right\rangle\langle\langle\psi \mid \psi\rangle\right.$.

The collocation matrix of the Hamiltonian operator on the original $\mathrm{N}$-grid is expressed in terms of a representation of the Hamiltonian operator on a finer $M$-grid. The eigenvalues of the $(N-1) \times(N-1)$ Hamiltonian matrix are exact upper bounds only when $M \rightarrow \infty$. Clearly, the computational cost of using this improved matrix instead of the original one discussed in the preceding section depends on the size of the hidden grid (i.e. on $M$ ).

The idea of using more grid points than spectral basis functions within a collocation approach is not new. For example, we mention the works of Fresnier [37], and more recently of Corey and Lemoine [38] and Corey and Tromp [39]. In particular our equation (20) with the approximation (22) is similar to Eq. (14) of Ref. [39], where however the resulting matrix is a $(M-1) \times(M-1)$ matrix with $M-N$ vanishing eigenvalues. More precisely, both equations are equivalent only in the case of a uniform grid. In fact, the approach followed by Corey and Tromp essentially consists of evaluating the kinetic-energy operator on the coarser grid and the potential part on the finer grid. In the special case of a uniform grid, i.e. $\rho_{g}(x)=1$, the kineticenergy operator has an exact LSF $(N-1) \times(N-1)$ matrix representation on the grid and its projection onto the finer grid is exact. Under such conditions the two approaches are equivalent.

On the other hand, in more general cases the matrix elements of the kinetic-energy operator involve matrix elements of the density (either the grid density, or a physical density, or a conformal density) and therefore the two approaches are not equivalent. A third approach, which can also be used in these cases, consists of expressing the operators $\hat{S}$ on the coarser grid, because its representation is exact, and only expressing the remaining matrix elements on the finer grid. We will compare these alternative approaches later on.

It is worth noting that in any of these approaches, independently of the size of the finer grid (as long as $M>N$ ), the matrices obtained with the collocation are not sparse, a feature which considerably increases the memory requirements for large grids.

\section{Applications}

In this section we consider some selected applications of the $v$ LSF.

\subsection{Strings of variable density}

We first consider the application of present approach to the Helmholtz equation for an inhomogeneous string of length $2 L$ :

$$
-\frac{d^{2}}{d x^{2}} \Psi_{n}(x)=E_{n} \rho(x) \Psi_{n}(x)
$$

where $E_{n}$ are the eigenvalues, $\rho(x)>0$ is the density of the string and we assume that the eigenfunctions $\Psi_{n}(x)$ satisfy Dirichlet boundary conditions $\Psi_{n}( \pm L)=0$.

On defining $\Phi_{n}(x) \equiv \sqrt{\rho(x)} \Psi_{n}(x)$ we obtain the equivalent equation [21]

$$
\hat{O} \Phi_{n}(x)=-\frac{1}{\sqrt{\rho(x)}} \frac{d^{2}}{d x^{2}} \frac{1}{\sqrt{\rho(x)}} \Phi_{n}(x)=E_{n} \Phi_{n}(x)
$$

The collocation approach discussed in the preceding sections can now be straightforwardly applied to this problem. 
As we have seen, the discretization of the problem may use an arbitrary positive definite density, which here we call $\rho_{g}(x)$, which determines the distribution of the collocation points on the string. This function does not need to be the physical density of the string, $\rho(x)$, although we will see that the case $\rho_{g}(x)=\rho(x)$ exhibits important computational advantages for the calculation of highly excited states.

The matrix elements of the hermitian operator appearing in the eigenvalue equation are easily obtained as

$$
\begin{aligned}
& O_{k l}=\left[-\frac{1}{\sqrt{\rho(x)}} \frac{d^{2}}{d x^{2}} \frac{1}{\sqrt{\rho(x)}}\right]_{k l} \\
& =\frac{1}{\sqrt{\rho\left(x_{k}\right)}}\left[\sqrt{\rho_{g}\left(x_{k}\right)} S_{k l} \sqrt{\rho_{g}\left(x_{l}\right)}+D_{k l}\right] \frac{1}{\sqrt{\rho\left(x_{l}\right)}},(24)
\end{aligned}
$$

where $x_{k}(k=1, \ldots, N-1)$ are the grid points corresponding to $\rho_{g}(x)$. In principle one has to calculate $N(N-1) / 2$ elements of the symmetric matrix for $\hat{O}$. The collocation matrices for $D(x)$ and for the densities on the grid are diagonal so that we need the $N-1$ elements for each of them. The collocation matrix for the operator $\hat{S}$ is not sparse but it is universal and, consequently, it is convenient to calculate and store it for later use in all the problems of the same kind. These properties make our collocation method highly flexible, allowing one to study problems of different density and using grids with different distributions of points without having to recalculate all the matrix elements.

Obviously, the choice of a suitable grid may lead to accurate results with a smaller number of grid points. We have found that the "natural density" $\rho_{g}(x)=\rho(x)$ is convenient for the highly excited states of the string. For $n \rightarrow \infty$ the WKB wavefunctions tend to become eigenfunctions of the operator $\hat{O}$ and therefore a discretization based on these functions is expected to be successful. On the other hand, if one is interested in the fundamental mode of the string, the optimal distribution of grid points should be determined by a grid density $\rho_{g}(x)$ that leads to a minimal Rayleigh quotient for the fundamental WKB eigenfunction corresponding to this density. In the special case $D(x)=0$ (the WKB wavefunctions are exact) the natural grid also minimizes the Rayleigh quotient.

We will illustrate these aspects of our collocation approach by means of the specific example of inhomogeneous string, which was first studied by Horgan and Chan [29]:

$$
\rho(x)=\frac{(\alpha+2)^{2}}{4\left(\alpha(\alpha+2)\left(x+\frac{1}{2}\right)+1\right)}
$$

where $|x| \leq 1 / 2$. These authors have shown that the frequencies of this string are given by $\omega=\alpha \lambda$ where $\lambda$ is a zero of the transcendental equation

$$
J_{1}(\lambda) Y_{1}((\alpha+1) \lambda)-Y_{1}(\lambda) J_{1}((\alpha+1) \lambda)=0
$$

and $J$ and $Y$ are the well known Bessel functions of first and second kind.

Fig. 1 shows the error $\equiv \equiv\left|1-E_{n}^{(\text {num })}\right| E_{n}^{(\text {exact })} \mid$ for the first 2499 states of that string with $\alpha=1$. The solid and dashed lines are respectively given by $N=2500$ points distributed according to the natural density and the uniform grid. The accuracy of the numerical results obtained with the natural grid are mostly of the order of the 12 digits-precision used in the calculation (hence the large plateaux).

Present LSF-collocation results for large $n$ are useful for the numerical calculation of the coefficients of the asymptotic expansion of the energy of the string, which for an arbitrary density is of the form

$$
E_{n} \approx A_{1} n^{2}+A_{2}+A_{3} / n^{2}+\ldots
$$

There are explicit formulas for the first three coefficients and Amore [21] has recently derived a general formula for $A_{3}$ in terms of an infinite series. This author has also calculated the first 5 coefficients $A_{j}$ numerically for the density (25) with $\alpha=1$ by fitting the expansion (26) to the first 10000 energies of the string calculated with 200digits precision. The three leading coefficients estimated in this way are in perfect agreement with the theoretical results given by well-known explicit formulas.

By straightforwardly fitting the asymptotic formula (26) to the first 2499 energies calculated numerically with the natural grid and $N=2500$ we found

$$
\begin{aligned}
E_{n} & \approx 9.869604401 n^{2}+0.375027633 \\
& -0.032450258 / n^{2}+0.005933450 / n^{4} .
\end{aligned}
$$

This result compares quite favorably with the formula obtained using the extrapolated values:

$$
\begin{aligned}
E_{n} & =9.869604401 n^{2}+0.375 \\
& -\frac{0.03265233457}{n^{2}}+\frac{0.009170584905}{n^{4}}+\ldots
\end{aligned}
$$




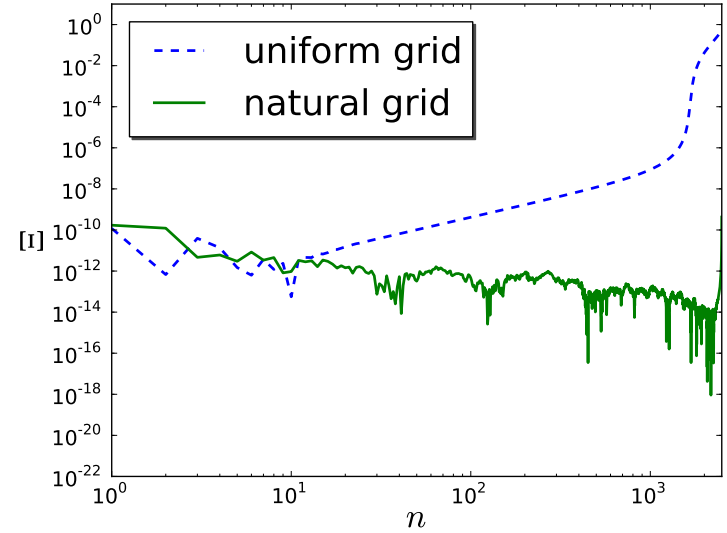

Figure 1. Relative error $\equiv \equiv\left|1-E_{n}^{(\text {num })} / E_{n}^{(\text {exact })}\right|$ for the first 2499 states of the string of Horgan and Chan [29] with $\alpha=1$. The solid line corresponds to a grid with $N=2500$ and its points distributed according to the natural density. The dashed line corresponds to a uniform grid with the same number of points.

\subsection{Circular membrane}

The conformal collocation method (CCM) devised elsewhere $[22,28]$ can be used to obtain precise numerical approximations to the eigenvalues and eigenfunctions of the Helmholtz equation on arbitrary domains in two di- mensions. This method has been applied to a wide range of domains: to the circle and to a circular waveguide with circular ridges [28], to the cardioid and to various regular polygons [22], to circular and asymmetric annuli [30], and to an inhomogeneous cardioid [31]). Here we would like to apply the present approach to a uniform circular membrane of unit radius which was earlier treated by means of a uniform grid [28]. Our purpose is to study the effect of an inhomogeneous grid on the precision of the results and on their rate of convergence towards the exact results.

For present numerical experiment we choose

$$
\rho_{g}(x)=\alpha \operatorname{csch}^{2}(\sqrt{\alpha}) \cosh ^{2}(\sqrt{\alpha} x)
$$

where $\alpha$ is a real parameter (note that the resulting grid is uniform when $\alpha=0$ ). Correspondingly we have

$$
\sigma(x)=1+\operatorname{csch}(\sqrt{\alpha}) \sinh (\sqrt{\alpha} x) .
$$

By means of the map from the circle to the square explicitly given in Eq. (18) of Ref. [28] we convert the original Helmholtz equation into an equivalent Helmoltz equation for a square membrane with a "conformal density" $\Sigma(x, y)$. Correspondingly the representation of this equation on the grid is

$$
\begin{aligned}
\hat{O}_{k_{1}, k_{2} ; l_{1}, l_{2}} & =\frac{1}{\sqrt{\sum\left(x_{k_{1}}, y_{k_{2}}\right)}}[-\Delta]_{k_{1}, k_{2} ; l_{1}, l_{2}} \frac{1}{\sqrt{\sum\left(x_{l_{1}}, y l_{2}\right)}} \\
& =\frac{1}{\sqrt{\sum\left(x_{l_{1}}, y l_{2}\right)}}\left\{\left[\sqrt{\rho\left(x_{k_{1}}\right)} S_{k_{1} l_{1}} \sqrt{\rho\left(x_{l_{1}}\right)}+D_{k_{1} l_{1}}\right] \delta_{k_{2} l_{2}}\right. \\
& \left.+\left[\sqrt{\rho\left(y_{k_{2}}\right)} S_{k_{2} l_{2}} \sqrt{\rho\left(y l_{2}\right)}+D_{k_{2} l_{2}}\right] \delta_{k_{1} l_{1}}\right\} \frac{1}{\sqrt{\sum\left(x_{l_{1}}, y l_{2}\right)}} .
\end{aligned}
$$

This expression approximates the matrix elements of the operator $\hat{O}=-\frac{1}{\sqrt{\Sigma(x)}} \Delta \frac{1}{\sqrt{\Sigma(x)}}$ connecting a point $\left(x_{k_{1}}, x_{k_{2}}\right)$ to a point $\left(x_{l_{1}}, x_{l_{2}}\right)$. Labelling every point on the grid with a single integer ranging from 1 to $(N-1)^{2}$ one is able to obtain an explicit matrix representation for the operator on the grid. The eigenvalues of this matrix are approximations to the exact eigenvalues of the circular membrane.

Table 1 shows the lowest eigenvalue of the collocation matrix obtained with grids of different size and different values of the parameter $\alpha$ which controls the distribution of the grid points. Present results for $\alpha=0$ and $N \leq 80$ correspond to those obtained earlier with a uniform grid [28]. It is worth noticing that the collocation eigenvalues for the largest density deformation $\alpha=4$ seem to converge to the exact result from below. The greatest rate of convergence is obtained for $\alpha=3.2$ though the results for the two largest grids seem to oscillate probably due to round-off errors. In all the cases considered here the rate of convergence seems to be compatible with the asymptotic behavior $1 / \mathrm{N}^{4}$ already observed in earlier calculations with the uniform grid [28]. It follows from Table 1 that the rate of convergence is considerably larger when 


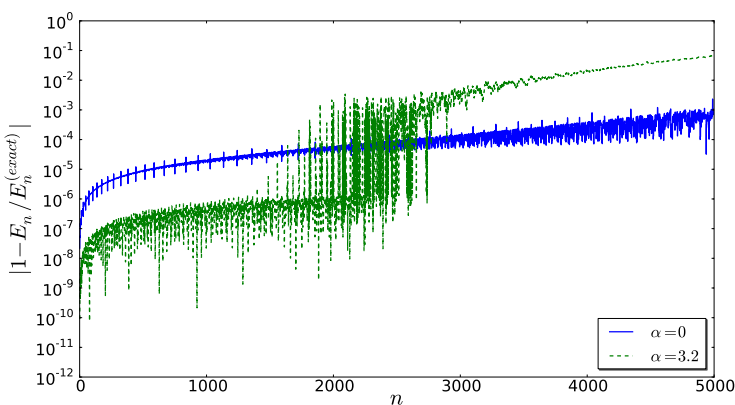

Figure 2. Relative error $\left|1-E_{n}^{(\text {num })} / E_{n}^{(\text {exact })}\right|$ for the first 5000 states of a circular drum of unit radius calculated on a grid with $N=120$ and $\alpha=0$ (solid line) and $\alpha=3.2$ (dashed line).

one resorts to a deformed grid density. For example, the result for $N=20$ and $\alpha=3.2$ is as accurate as the result for $N=120$ and $\alpha=0$.

Fig. 2 shows the relative error for the first 5000 eigenvalues for grids with $N=120$ and $\alpha=0$ (solid line) and $\alpha=3.2$ (dashed line). The deformed grid yields far more accurate results for the first 2000 states (except for isolated exceptions) whereas the uniform grid is preferable for $2000<n \leq 5000$ with its error increasing gently with $n$. This curious behavior has a simple explanation: the collocation spectrum for $\alpha=3.2$ is not linear for $n>2000$, thus departing from the behavior expected on the grounds of Weyl's law. On the other hand, the collocation spectrum for the uniform grid remains linear even for the highest excited states considered here. The reason is that in the case of the deformed grid more collocation points are moved towards the border of the region and for moderately excited states there is a considerable gain in resolution in the region where the solutions are more important, while retaining an acceptable resolution in the central region. However, for the highest excited states in the plot, the loss of resolution in the central part of the collocation region affects the overall quality of the results. No such effect takes place in the case of a uniform grid where the collocation region is covered more uniformly.

\subsection{Bound states in the continuum: crossed wires}

The present approach is not restricted to bounded domains as previous applications may suggest. The LSF collocation method is suitable for the treatment of open domains as shown in the following example. The configuration of orthogonal crossed wires was originally studied by Schult et al. [32] and later by Avishai et al. [33]. More recently, Bulgakov et al. [34] studied scissor-shaped waveguides; i.e. configurations with non-orthogonal arms.

Interestingly, Schult et al. discovered that despite the infinite extension of the cross with Dirichlet boundary conditions at the border, the waveguide just mentioned supports bound states. In particular, it was found that the fundamental mode of this waveguide falls below the continuum threshold, $E_{1}=0.66 E_{t}\left(E_{t}=\hbar^{2} \pi^{2} / 2 m w^{2}\right)$, and that a second bound state is embedded in the continuum: $E_{2}=3.72 E_{t}$. Those results were confirmed by Avishai et al. [33].

In order to apply our technique to this problem we resort to the conformal map which maps the unit disk onto the infinite cross as shown in problem 6, page 197 in Nehari's book [36]. Note that the there is a typo in that expression; the correct map from the unit circle to a cross with arms of width $w=2$ is:

$$
\begin{aligned}
f(z) & =\frac{\sqrt{2} \Gamma(1 / 4)}{\pi \Gamma(5 / 4)} \int_{0}^{z} \frac{\sqrt{1+z^{\prime 4}}}{\left(1-z^{\prime 4}\right)} d z^{\prime} \\
& =\frac{\sqrt{2} \Gamma(1 / 4)}{\pi \Gamma(5 / 4)} z F_{1}\left(\frac{1}{4} ;-\frac{1}{2}, 1 ; \frac{5}{4} ;-z^{4}, z^{4}\right)
\end{aligned}
$$

where $F_{1}$ is the Appell hypergeometric function. After composing this map with the map from the square to the unit circle, one obtains the map from the square to the cross, which is the one needed by our collocation method. Therefore, using this conformal map, we transform the homogeneous Helmholtz equation on the infinite cross into an inhomogeneous Helmholtz equation on the square, with a (conformal) density directly given in terms of the map. The left and right panels in Fig. 3 display the square of side 2 and its image, an infinite cross with arms of width $w=2$, respectively. The uniform grid on the square (with $N=20$ ) is mapped onto a deformed grid on the unit cross. The procedure for the collocation of the Hamiltonian operator on the grid is similar to the one above for the circle and we think it unnecessary to show the steps explicitly here.

Fig. 4 shows the ratio $E_{1} / E_{t}$ for grids obtained with the density (27) for different values of $\alpha$. The case $\alpha=0$ corresponds to the uniform grid shown in the left panel of Fig. 3, while larger values of $\alpha$ correspond to non-uniform grids where the points are progressively moved towards the extremities of the intervals.

Fig. 4 clearly shows that for fixed $N$ the eigenvalue as a function of $\alpha$ does not exhibit a minimum. We also appreciate that the value $\alpha \approx 2$ of the density parameter is nearly optimal. The first observation illustrates the fact that our pseudospectral approach does not obey the variational principle for finite $N$ (although it should be satisfied 
Table 1. The energy of the fundamental mode of a circular membrane calculated with the density (27) for different values of $\alpha$. The exact result is $E_{1}=\left(j_{0,1}\right)^{2} \approx 5.7831859629467$. The underlined digits are correct.

\begin{tabular}{|c|c|c|c|c|c|}
\hline$N$ & $\alpha=0$ & $\alpha=2$ & $\alpha=3$ & $\alpha=3.2$ & $\alpha=4$ \\
\hline 10 & $\underline{5.785633618939}$ & $\underline{5.783316758453}$ & $\underline{5.783980548966}$ & $\underline{5.78} 4734711375$ & 5.795622183490 \\
\hline 20 & $\underline{5.783} 347847095$ & $\underline{5.783194258510}$ & $\underline{5.783186788303}$ & $\underline{5.783186103068}$ & $\underline{5.783189070233}$ \\
\hline 30 & $\underline{5.783} 218252759$ & $\underline{5.783187596925}$ & $\underline{5.783186118919}$ & $\underline{5.783185972125}$ & $\underline{5.783185613788}$ \\
\hline 40 & $\underline{5.783196213318}$ & $\underline{5.783186479426}$ & $\underline{5.783186011997}$ & $\underline{5.783185965565}$ & $\underline{5.783185851816}$ \\
\hline 50 & $\underline{5.783190167777}$ & $\underline{5.783186174397}$ & $\underline{5.783185982981}$ & $\underline{5.783185963965}$ & $\underline{5.783185917378}$ \\
\hline 60 & $\underline{5.783187992366}$ & $\underline{5.783186064893}$ & $\underline{5.783185972593}$ & $\underline{5.783185963423}$ & $\underline{5.783185940958}$ \\
\hline 70 & $\underline{5.783187058902}$ & $\underline{5.783186017966}$ & $\underline{5.783185968148}$ & $\underline{5.783185963199}$ & $\underline{5.783185951073}$ \\
\hline 80 & $\underline{5.78318} 6605573$ & $\underline{5.783185995194}$ & $\underline{5.783185965994}$ & $\underline{5.783185963092}$ & $\underline{5.783185955985}$ \\
\hline 90 & $\underline{5.783186364220}$ & $\underline{5.783185983077}$ & $\underline{5.78318596} 4848$ & $\underline{5.783185963037}$ & $\underline{5.783185958599}$ \\
\hline 100 & $\underline{5.783186226262}$ & $\underline{5.783185976154}$ & $\underline{5.783185964194}$ & $\underline{5.783185963006}$ & $\underline{5.783185960094}$ \\
\hline 110 & $\underline{5.783186142775}$ & $\underline{5.783185972288}$ & $\underline{5.783185963856}$ & $\underline{5.783185963077}$ & $\underline{5.783185961467}$ \\
\hline 120 & $\underline{5.783186089902}$ & 5.783185969449 & 5.783185963555 & 5.783185962428 & 5.783185961492 \\
\hline
\end{tabular}
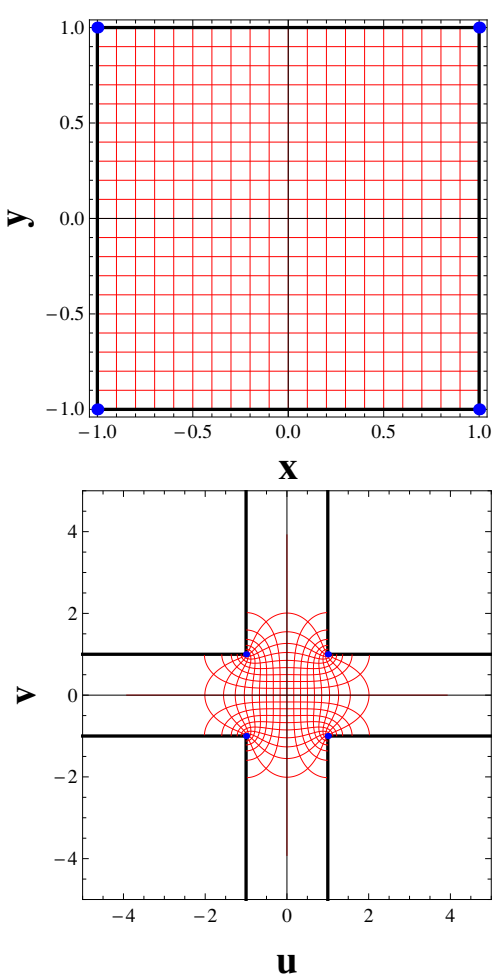

Figure 3. Square of side 2 and infinite cross of width 2 obtained with the conformal map. The red lines correspond to a grid with $N=20$.

in the limit $N \rightarrow \infty$ ). For this reason, the sequences of results with $\alpha \leq 2$ are monotonically decreasing, while, on the other hand, those with $\alpha \geq 2.1$ have positive slopes for small grids. For large enough grids, however, one should obtain a negative slope even for these densities. This is in fact the case for the values corresponding to $\alpha=2.1$, which start to decay for $N \geq 40$, although this behavior cannot be appreciated on the scale of the plot. The origin of this violation of the variational principle arises from the fact that we have not calculated the integrals explicitly, and that for large enough $\alpha$ the grid points are mostly concentrated at the corners of the square, which leads to imprecise approximations. Although one cannot resort to the variational principle or the PMS to determine the optimal $\alpha$ in this case, it is possible to obtain a reasonable value of $\alpha$ by looking for values of $\alpha$ which do not produce large changes in the energy when more grid points are added. Such behavior is a sound sign that the grid sampling is good. It is worth noticing that the use of nonuniform grid greatly improves the results. For example, the results for $\alpha=0$ and $N=100$ are considerably less accurate than those given by a coarser grid $(N=20)$ with a nearly optimal $\alpha$-value $(\alpha=2$ or $\alpha=2.1)$.

In Table 2 we display the values plotted in Fig. 4. The best set of results for the optimal value $\alpha=2.1$ provides an approximate upper bound $E_{1} / E_{t} \leq 0.659611$ for $N=120$. Although we cannot prove that this bound is rigorous (because as argued above the variational principle is obeyed only for $N \rightarrow \infty$ ) experience tells us that the sequence of collocation estimates decreases monotonically with $N$ for fine enough grids.

We have also carried out the improved collocation calculation described above for $\alpha=0$ (uniform grid) with matrix grids ranging from $N=8$ to $N=48$ and the auxiliary grid with $M=N+2$ (the rather low-order calculation is due to the fact the matrix is no longer sparse which increases the allocation-memory requirement). A straightforward fit of the results for the lowest eigenvalue to the formula $E_{1} / E_{t}(N)=c_{0}+c_{1} / N+c_{2} / N^{2}$ provides 


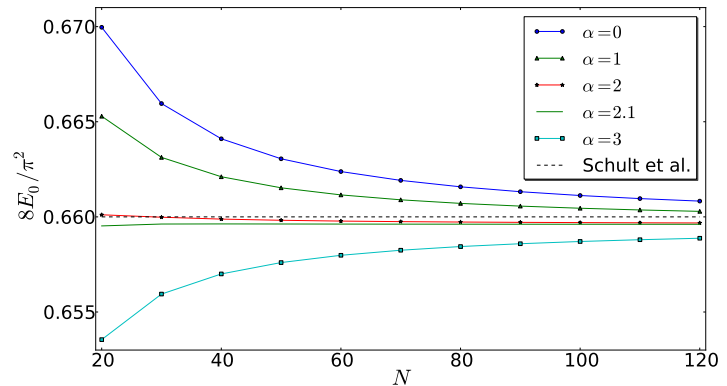

Figure 4. $8 E_{1} / \pi^{2}$ for grids obtained with the density (27) for different values of $\alpha$.

Table 2. Ratio $E_{1} / E_{t}$ for grids obtained with the density (27) for different values of $\alpha$.

\begin{tabular}{cccccc}
\hline$N$ & $\alpha=0$ & $\alpha=1$ & $\alpha=2$ & $\alpha=2.1$ & $\alpha=3$ \\
\hline \hline 20 & 0.669964 & 0.665289 & 0.660110 & 0.659525 & 0.653549 \\
30 & 0.665956 & 0.663127 & 0.659982 & 0.659624 & 0.655943 \\
40 & 0.664105 & 0.662109 & 0.659883 & 0.659630 & 0.657001 \\
50 & 0.663053 & 0.661528 & 0.659821 & 0.659626 & 0.657599 \\
60 & 0.662382 & 0.661155 & 0.659779 & 0.659622 & 0.657981 \\
70 & 0.661918 & 0.660897 & 0.659750 & 0.659618 & 0.658247 \\
80 & 0.661580 & 0.660709 & 0.659729 & 0.659616 & 0.658441 \\
90 & 0.661323 & 0.660566 & 0.659712 & 0.659614 & 0.658589 \\
100 & 0.661123 & 0.660454 & 0.659700 & 0.659613 & 0.658706 \\
110 & 0.660961 & 0.660364 & 0.659689 & 0.659612 & 0.658800 \\
120 & 0.660829 & 0.660290 & 0.659681 & 0.659611 & 0.658877
\end{tabular}

the estimate $c_{0} \approx 0.659617$ with is close to the result obtained with the standard approach. For this reason we think that our result is more accurate than the one obtained by Schult et al. [32].

More recently, Trefethen and Betcke [35] obtained an improved estimation of the second bound state found earlier by Schult et al:: $E_{2} / E_{t}=3.71648$. We have carried out a collocation calculation with $\alpha=1$ and $N$ ranging from 70 to 100 and obtained $E_{2} / E_{t}=3.72797$ for the finest grid. A least square fit of such data to the function $\left[E_{2} / E_{t}\right](N)=c_{0}+c_{1} / N+c_{2} / N^{2}$ yielded the more accurate estimate $E_{2} / E_{t} \approx 3.7161$ in better agreement with the result of Trefethen and Betcke [35].

\section{Conclusions}

The accuracy of a pseudospectral (collocation) method may be greatly enhanced using a suitable mapping of the coordinates, in one or more dimensions. We have shown that this map can be chosen using the PMS. For example, the application of this procedure to the case of a crossshaped membrane with infinite orthogonal arms confirms the well-known result that the fundamental mode of this system is bound and we were able to calculate its energy with high accuracy.

From a computational point of view the present method is both efficient and highly flexible. It allows one to express the collocation matrix for a given problem in terms of a "heavy" universal matrix for the laplacian on the grid, which can be calculated once and stored, and of a "light" diagonal matrix, which is specific to the problem and can be calculated more rapidly.

\section{Acknowledgements}

P.A. acknowledges support of Conacyt through Sistema Nacional de Investigadores (SNI).

\section{References}

[1] J.P. Boyd, Chebyshev and Fourier spectral methods (Dover, 2000)

[2] G. Yao, S.I. Chu, Chem. Phys. Lett. 204, 381 (1993)

[3] J. Wang, S.I. Chu, C. Laughlin, Phys. Rev. A 50, 3208 (1994)

[4] E. Fattal, R. Baer, R. Kosloff, Phys. Rev. E 53, 1217 (1996)

[5] H. Wei, J. Chem. Phys. 106, 6885 (1997)

[6] B. Poirier, J.C. Light, J. Chem. Phys. 111, 4869 (1999)

[7] D. Baye, M. Hesse, M. Vincke, Phys. Rev. E 65, 026701 (2002)

[8] R.G. Littlejohn, M. Cargo, J. Chem. Phys. 116, 8691 (2002)

[9] A.K. Roy, S.I. Chu, Phys. Rev. E 65, 052508 (2002)

[10] J.P. Boyd, C. Rangan, P.H. Bucksbaum, J. Comp. Phys. 188, 56 (2003)

[11] P.M. Stevenson, Phys. Rev. D 23, 2916 (1981)

[12] A. Okopińska, Phys. Rev. D 35, 1835 (1997)

[13] A. Duncan, M. Moshe, Phys. Lett. B 215, 352 (1988)

[14] G. Krein, D.P. Menezes, M. B. Pinto, Phys. Lett. B 370, 5 (1996)

[15] M.P. Blencowe, A.P. Korte, Phys. Rev. B 56, 9422 (1997)

[16] M.B. Pinto, R.O. Ramos, Phys. Rev. D 60, 105005 (1999)

[17] H.F. Jones, P. Parkin, D. Winder, Phys. Rev. D 630 125013 (2001)

[18] J.L. Kneur, M.B. Pinto, R.O. Ramos, Phys. Rev. Lett. 89, 210403 (2002) 
[19] P. Amore, M. Cervantes, F.M. Fernández, J. Phys. A 40, 13047 (2007)

[20] C.M. Bender, S.A. Orszag, Advanced mathematical methods for scientists and engineers (McGraw-Hill, 1978)

[21] P. Amore, Annals of Phys. 326, 2315 (2011)

[22] P. Amore, J. Math. Phys. 51, 052105 (2010)

[23] P. Amore, Annals of Phys. 325, 2679 (2010)

[24] G. Borg, Acta Math. 78, 1 (1946)

[25] H.P.W. Gottlieb, Inverse Problems 18, 971 (2002)

[26] J.W.S. Rayleigh, The Theory of Sound vol. 1 (New York: Dover, 1945)

[27] R. Guardiola, F.M. Fernández, Eur. Phys. J. D 21, 19 (2002)

[28] P. Amore, J. Phys. A 41, 265206 (2008)

[29] C.O. Horgan, A.M. Chan, Journal of Sound and Vibration 225, 503 (1999)

[30] C. Alvarado, P. Amore, J. Math. Phys. 52, 063516
(2011)

[31] P. Amore, Europhys. Lett. 92, 10006 (2010)

[32] R.L. Schult, D.G. Ravenall, H.W. Wyld, Phys. Rev. B 39, 5476 (1989)

[33] Y. Avishai, D. Bessis, B.G. Giraud, G. Mantica, Phys. Rev. B 44, 8028 (1989)

[34] E.N. Bulgakov, P. Exner, K.N. Pichugin, A.F. Sadreev, Phys. Rev. B 66, 155109 (2002)

[35] L.N. Trefethen, T. Betcke, Contemp. Math. 412, 297 (2006)

[36] Z. Nehari, Conformal mapping (McGraw-Hill, 1952)

[37] R.A. Fresnier, J. Chem. Phys. 85, 1462 (1986)

[38] G.C. Corey, D. Lemoine, J. Chem. Phys. 97, 4115 (1992)

[39] G.C. Corey, J.W.Tromp, J. Chem. Phys. 103, 1812 (1995) 